\title{
Rigorous Quantitative SEM/EDS Microanalysis Requires Careful Inspection of the Peak-Fitting Residual Spectrum to Reveal Hidden Constituents
}

\author{
Dale E. Newbury ${ }^{1}$ and Nicholas W. M. Ritchie ${ }^{1}$ \\ 1. National Institute of Standards and Technology, Gaithersburg, MD
}

Electron-excited x-ray microanalysis performed with the scanning electron microscope and energy dispersive $\mathrm{x}$-ray spectrometry (SEM/EDS) has been shown to be capable of the same degree of accuracy as achieved with high spectral resolution wavelength dispersive spectrometry, even when severe peak interferences occur [1]. Achieving this level of analytical performance on a specimen with a complex composition requires a careful strategy of alternating qualitative and quantitative analyses. While major constituents can usually be recognized during the initial qualitative analysis, minor and trace constituents that suffer interference from the major constituent peaks are likely to be missed. To reliably detect such minor and trace constituents, and even major constituents in certain severe interference situations, the quantitative analysis software must construct the "residual spectrum" that remains after peak fitting has removed all of the characteristic x-ray peaks of the identified constituents. This residual spectrum is then examined, and if new constituents are discovered, then the quantitative analysis is repeated. For complex interference situations, it may be necessary to perform several rounds of alternating qualitative and quantitative analyses.

An example of this procedure is illustrated by the analysis of a monazite grain found in a sample of Kalimantan sand. The EDS spectrum contained 48.8 million counts accumulated at $8 \%$ deadtime with 20 $\mathrm{keV}$ beam energy. The initial qualitative analysis revealed major $\mathrm{O}, \mathrm{P}, \mathrm{La}$, and $\mathrm{Ce}$, with minor to trace $\mathrm{Al}, \mathrm{Si}, \mathrm{Ca}$, and Th. After peak fitting for these elements using NIST DTSA II [2], the residual spectrum shown in Figure 1 revealed additional peaks for $\mathrm{Ti}, \mathrm{Nd}$ and $\mathrm{Pr}$ hidden beneath the $\mathrm{La}$ and $\mathrm{Ce}$ families (brown residual). A second round of peak fitting including these elements gave the blue residual spectrum, qualitative analysis of which revealed Sm and Fe. Including all of these elements gave the final residual spectrum (green trace). Further exploration of this rare earth L-family region for other rare earths or transition elements at even lower concentrations would require accumulation of more counts. A similar sequence of analyses in the region of the $\mathrm{P}$ K-family is shown in Figure 2. After the initial peak fitting for $\mathrm{Si}$ and $\mathrm{P}$ (brown residual), Sr and $\mathrm{Zr}$ were identified. After further fitting, $\mathrm{Y}$ and $\mathrm{Nb}$ were identified (green residual), and fitting for all constituents yielded the final residual (blue). Again, further analysis would require accumulation of more counts. The quantitative results found in this sequence of three analyses are presented in Table 1 (with oxygen calculated by assumed stoichiometry). Note the progression of the raw analytical total toward unity as the hidden constituents are successively discovered and included in the quantitative analysis. If normalization was applied after the first analysis round and the residual spectrum was unavailable for inspection, the minor/trace constituents suffering interference would likely be missed.

\section{References:}

[1] D. Newbury and N. Ritchie, J. Materials Sci., 50 (2015) 493-518.

[2] N. Ritchie, DTSA-II available free at: www.cstl.nist.gov/div837/837.02/epq/dtsa2/index.html 
Table 1. Analysis of a Monazite grain (raw mass concentrations).

\begin{tabular}{|c|c|c|c|c|c|c|}
\hline Round & $\begin{array}{l}\text { O (by assumed } \\
\text { stoichiometry) }\end{array}$ & Al & Si & $\mathbf{P}$ & $\mathrm{Ca}$ & $\mathrm{Ti}$ \\
\hline First analysis & $0.2363 \pm 0.0008$ & $0.0013 \pm 0.0000$ & $0.0049 \pm 0.0000$ & $0.1114 \pm 0.0006$ & $0.0007 \pm 0.0000$ & \\
\hline Second analysis & $0.2828 \pm 0.0009$ & $0.0016 \pm 0.0000$ & $0.0059 \pm 0.0001$ & $0.1240 \pm 0.0007$ & $0.0007 \pm 0.0000$ & $0.0071 \pm 0.0001$ \\
\hline Third analysis & $0.2908 \pm 0.0010$ & $0.0015 \pm 0.0000$ & $0.0061 \pm 0.0001$ & $0.1263 \pm 0.0007$ & $0.0007 \pm 0.0000$ & $0.0072 \pm 0.0001$ \\
\hline Iteration & $\mathrm{Fe}$ & $\mathrm{Sr}$ & $\mathbf{Y}$ & $\mathrm{Zr}$ & Nb & La \\
\hline First analysis & & & & & & $0.1359 \pm 0.0002$ \\
\hline Second analysis & & $0.0016 \pm 0.0001$ & $0.0098 \pm 0.0002$ & $0.0113 \pm 0.0002$ & & $0.1585 \pm 0.0003$ \\
\hline Third analysis & $0.0022 \pm 0.0001$ & $0.0028 \pm 0.0001$ & $0.0030 \pm 0.0002$ & $0.0117 \pm 0.0002$ & $0.0006 \pm 0.0001$ & $0.1591 \pm 0.0003$ \\
\hline Iteration & $\mathrm{Ce}$ & $\mathrm{Pr}$ & Nd & Sm & Th & Raw Sum \\
\hline First analysis & $0.2692 \pm 0.0004$ & & & & $0.0038 \pm 0.0001$ & $0.7635 \pm 0.0011$ \\
\hline Second analysis & $0.2699 \pm 0.0004$ & $0.0221 \pm 0.0003$ & $0.0750 \pm 0.0003$ & & $0.0040 \pm 0.0001$ & $0.9629 \pm 0.0013$ \\
\hline Third analysis & $0.2709 \pm 0.0004$ & $0.0221 \pm 0.0003$ & $0.0751 \pm 0.0003$ & $0.0073 \pm 0.0005$ & $0.0040 \pm 0.0001$ & $0.9960 \pm 0.0030$ \\
\hline
\end{tabular}
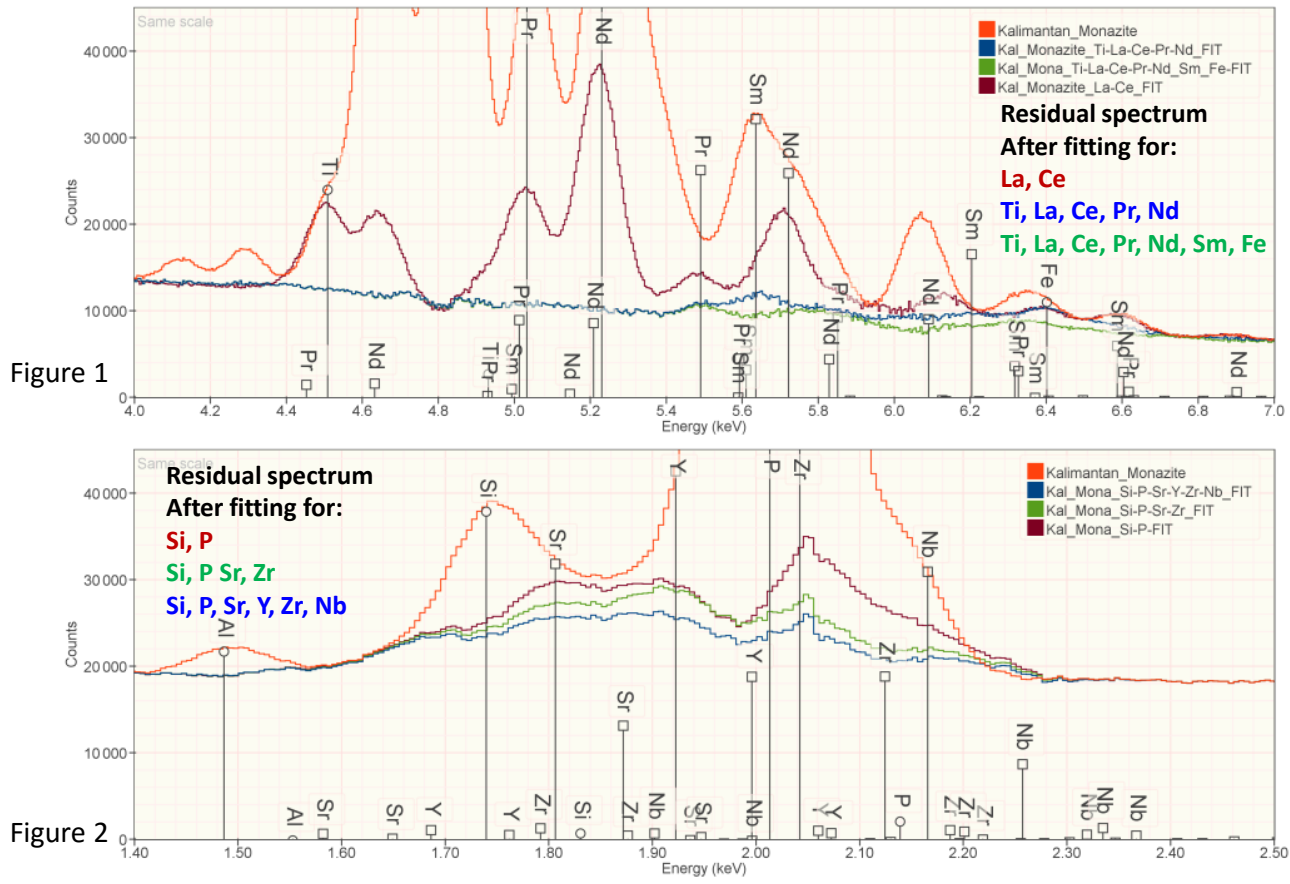

Figure 1. EDS spectrum of a monazite grain: rare earth element L-family region; $\mathrm{E}_{0}=20 \mathrm{keV}$. Figure 2. Phosphorus K-family region of the EDS spectrum of a monazite grain. 